\title{
The Collagen Challenge: Preventing Myocardial Fibrosis Is the Hype Reflective of the Potential?
}

\author{
John D Rozich* \\ Department of Medicine, Tuomey Hospital Sumter, USA
}

*Corresponding author: John D Rozich, Department of Medicine, Tuomey Hospital Sumter, USA.

Received Date: September 24, 2019

Published Date: October 01, 2019

\section{Mini Review}

Mounting evidence has suggested that the cardiac extracellular matrix (ECM) has a nuanced but determinative role in myocardial remodeling in response to pathological insult and subsequent recovery [1-3]. These alterations in the structure and actual composition of ECM may actually reflect many of the welldescribed phenotypic cardiac properties typical of common diseases states including diabetes, hypertension, obesity and ischemia. Current thought is that dysregulatory influences result in a maladaptive remodeling process that begins an inevitable progression to irreversible and often untimely cardiac failure $[1,2]$. This potentially injurious perturbation in the dysfunctional ECM response is complex involving profibrotic collagen synthesis and post-translational modification including increased crosslinking and reduced degradation $[1,4]$. The result is a stiffening noncompliant and impaired myocardium that underpins observed clinical changes causing the heart failure syndrome. Sampling changing serum levels of distinct proteins or molecular fragments are now being used to assess collagen turnover. This includes using the N-terminal propeptide of procollagen type III and C-terminal propeptide of procollagen type I considered directly correlative to histologically proven fibrosis $[1,5]$. Other biomarkers have been proposed to be associated with pathology involving collagen metabolism, (tissue inhibitor of metalloproteinases-1 (TIMP-1), matrix metalloproteinase [MMP]-2, MMP-9, galectin-3 [Gal-3], etc) but are not yet validated as directly representative of histologically confirmed fibrosis $[1,6]$.

Very recent publications have raised the intriguing possibility that a certain pharmacological class of drugs, exemplified by entresto [sacubitril/valsartan], may be antifibrotic linked to an improved response in examined cohorts [7-9]. There are clearly limitations with the data examined thus far that must temper any premature conclusive enthusiasm, but the potential of selectively influencing the adverse outcomes associated with myocardial hypertrophic growth is indeed remarkable. It begs the question as to whether cohorts shown to have disproportionate hypertrophy in the setting of previously mentioned chronic disease states should be explicitly targeted for use of entresto or like agents. Additionally, should different hemodynamic conditions causing excessive hypertrophic growth, including the pressure overloaded state of progressive aortic stenosis also be considered?Foreseeably, there are numerous remaining questions including whether the sacubitril/valsartan affect is mechanistically distinct from other agents, based on its combined (perhaps additive) molecular targets $[3,4,10]$. Alternatively is the collagen impact secondary to a family of drugs, including enalapril, (since a subset of the PARADIGMHF data were examined); all able to impact fibrosis but though mechanism(s) not presently understood [1,2,5,6]? Also under consideration is whether different forms of phenotypic hypertrophy, as dictated by different variables among hosts, demonstrate fundamentally different responsiveness. Keys to the prospect of enhanced specific therapy directed at reigning in the maladaptive fibrotic process are the following. First, clear evidence must emerge showing use of collagen biomarkers is an acceptable substitute to direct histopathological evidence of fibrosis in different forms of phenotypically expressed myocardial hypertrophy $[1,7,8]$. Not all forms of hypertrophy responsive to distinct triggers may respond uniformly even when appearing similar [11,12]. And existing literature is replete with false or minimally relevant surrogate indices creating disappointing time-consuming blind alleys [13].

Second, it will be important to address whether there are any important unanticipated factors impacting the serum levels reflecting collagen turnover [11]. This concern, while closely related to the first point, differs in a subtle but key aspect. If sacubitril/valsartan or other similar agents substantively alter 
or dynamically influence renal clearances for example, volume of distribution and bioavailability may also be impacted. Thus, assigning determinative insights to variations in referent serum levels may still reflect valid collagen turnover metrics, but imbedded adjustments will be requisite to ensure validity of global fibrotic impact. Finally, the entire evolving menu of serum biomarkers supporting collagen turnover must be individually examined in their response to other well-characterized nonfibrotic biomarkers to ensure that a form of "external quality control" exists. The use of BNP, troponin and N-terminal proBNP have reflected this need and are believed important, but other markers may demonstrate prescient relevance $[3,4]$. The demographics of aging, obesity, hypertension and diabetes reveal a growing threat of heart failure in its various forms $[14,15]$. The ability to slow the progression of fibrotic injury to the vulnerable myocardium will potentially be a significant tool but will require accompanying detail in the actual mechanisms of cardiac transformation from health to disease. Entering the "game" too late, for individuals or populations at risk may falsely demonstrate no real impact on the metrics of survival. But a temporal understanding of the natural progression of fibrosis and scar will undoubtedly raise hope for ideal intervention precluding emergence of the low output state as well as threatening dysrhythmias. Despite the gaps in existing knowledge, there is excitement in the air as clinicians begin to anticipate optimizing therapy linked to metrics of improved survival. Much work needs to be done but already there are populations at risk that some have proposed may disproportionately benefit from this therapy.

\section{Acknowledgement}

None.

\section{Conflict of Interest}

No conflict of interest.

\section{References}

1. Lopez B, Gonzalez A, Ravassa S, Beaumont J, Moreno MU, et al. (2015) Circulating Biomarkers of Myocardial Fibrosis: The Need for a Reappraisal. J Am Coll Cardiol 65(22): 2449-2456.

2. Condorelli G, Jotti GS, Pagiatakis C (2016) Fibroblast Senescence as a Therapeutic Target of Myocardial Fibrosis: Beyond Spironolactone? J Am Coll Cardiol 67(17): 2029-2031.
3. Zannad F, Ferreira JP (2019) Is Sacubitril/Valsartan Antifibrotic? J Am Coll Cardiol 73(7): 807-809.

4. Zile MR, O Meara E, Claggett B, Prescott MF, Solomon SD, et al. (2019) Effects of Sacubitril/Valsartan on Biomarkers of Extracellular Matrix Regulation in Patients With HFrEF. J Am Coll Cardiol 73(7): 795-806.

5. Heymans S, Gonzalez A, Pizard A, Papageorgiou AP, Lopez-Andres N, et al. (2015) Searching for new mechanisms of myocardial fibrosis with diagnostic and/or therapeutic potential. Eur J Heart Fail 17(8): 764-771.

6. Ferreira JM, Ferreira SM, Ferreira MJ, Falcao Pires I (2017) Circulating Biomarkers of Collagen Metabolism and Prognosis of Heart Failure with Reduced or Mid-Range Ejection Fraction. Curr Pharm Des 23(22): 32173223.

7. Iraqi W, Rossignol P, Angioi M, Fay R, Nuee J, et al. (2009) Extracellular cardiac matrix biomarkers in patients with acute myocardial infarction complicated by left ventricular dysfunction and heart failure: insights from the Eplerenone Post-Acute Myocardial Infarction Heart Failure Efficacy and Survival Study (EPHESUS) study. Circulation 119(18): 2471-2479.

8. Zannad F, Alla F, Dousset B, Perez A, Pitt B (2000) Limitation of excessive extracellular matrix turnover may contribute to survival benefit of spironolactone therapy in patients with congestive heart failure: insights from the randomized aldactone evaluation study (RALES). Rales Investigators. Circulation 102(22): 2700-2706.

9. Ferreira JP, Duarte K, Montalescot G, Pitt B, De Sa EL, et al. (2018) Effect of eplerenone on extracellular cardiac matrix biomarkers in patients with acute ST-elevation myocardial infarction without heart failure: insights from the randomized double-blind REMINDER Study. Clin Res Cardiol 107(1): 49-59.

10. Mc Murray JJ, Packer M, Desai AS, Gong J, Lefkowitz MP, et al. (2014) Angiotensin-neprilysin inhibition versus enalapril in heart failure. N Engl J Med 371(11): 993-1004.

11. Gerdts E, Roman MJ, Palmieri V, Wachtell K, Smith G, et al. (2004) Impact of age on left ventricular hypertrophy regression during antihypertensive treatment with losartan or atenolol (the LIFE study). J Hum Hypertens 18(6): 417-422.

12. Rudolph A, Abdel Aty H, Bohl S, Boye P, Zagrosek A, et al. (2009) Noninvasive detection of fibrosis applying contrast-enhanced cardiac magnetic resonance in different forms of left ventricular hypertrophy relation to remodeling. J Am Coll Cardiol 53(3): 284-291.

13. Fleming TR, DeMets DL (1996) Surrogate end points in clinical trials: are we being misled? Ann Intern Med 125(7): 605-613.

14. Sowers JR, Epstein M, Frohlich ED (2001) Diabetes, hypertension, and cardiovascular disease: an update. Hypertension 37(4): 1053-1059.

15. Johnson RJ, Segal MS, Sautin Y, Nakagawa T, Feig DI, et al. (2007) Potential role of sugar (fructose) in the epidemic of hypertension, obesity and the metabolic syndrome, diabetes, kidney disease, and cardiovascular disease. Am J Clin Nutr 86(4): 899-906. 